\title{
An Apparatus for Measurement of Thermal Conductivity of Solids at Low Temperatures
}

\author{
Robert L. Powell, William M. Rogers, and Don O. Coffin
}

\begin{abstract}
A description is given of an apparatus used for determining the thermal conductivities of solids in the temperature range $4^{\circ} \mathrm{K}$ to $300^{\circ} \mathrm{K}$. The apparatus is especially suited to the determination of thermal conductivity over a large temperature interval, enabling coverage of the temperatures between the normal boiling points of liquefied gases. Illustrative results are given for an insulator, polytetrafluoroethylene, and for a high-conductivity commercial coalesced copper. The thermal conductivity of the insulator increases monotonically from 0.56 milliwatt per centimeter per degree $\mathrm{K}$ at $5^{\circ} \mathrm{K}$, to 2.32 at $80^{\circ} \mathrm{K}$. The thermal conductivity of the copper has a maximum of 24.9 watts per centimeter per degree $\mathrm{K}$ at $21^{\circ} \mathrm{K}$, and a value of 8.15 at $5^{\circ} \mathrm{K}$. The methods of data analysis and estimation of errors are given.
\end{abstract}

\section{Introduction}

Data on thermal conductivity are necessary for the selection of suitable construction materials, and the prediction of operating characteristics of lowtemperature apparatus used in research and industry. In addition, accurate thermal conductivity values for pure metals and controlled alloys are important in the development and verification of theories of electronic transport phenomena in metals. The apparatus described in this paper is well suited to the measurement of thermal conductivity of metals and commercial alloys; it is also usable for poor conductors such as plastics and other dielectric solids.

\section{Experimental Apparatus}

\subsection{General Description}

The method of determining thermal conductivity by axial heat flow through a long cylindrical sample is used. A pictorial diagram of the apparatus is shown in figure 1. The apparatus is similar, in many respects, to some of those described in the review by Olsen and Rosenberg [5].

The cryostat consists of concentrically mounted sample, thermal shield, vacuum container, glass Dewar, Dewar support, and outside metal Dewar.

The sample rod is clamped to the heat sink at the top; the sample heater is attached to the bottom of this rod. The temperature distribution along the sample is measured by means of eight thermocouples attached to thermocouple holders positioned along the rod. Heat losses by gas convection and conduction are made negligible by evacuating the region surrounding the sample. Losses by radiation and conduction along the lead wires are reduced by enclosing the sample rod within a symmetric cylindrical thermal shield maintained at approximately the same temperature and axial gradient as the sample. Measurements of the temperature, thermal gradient,

1 Now at the Los Alamos Scientific Laboratory, Los Alamos, New Mexico.

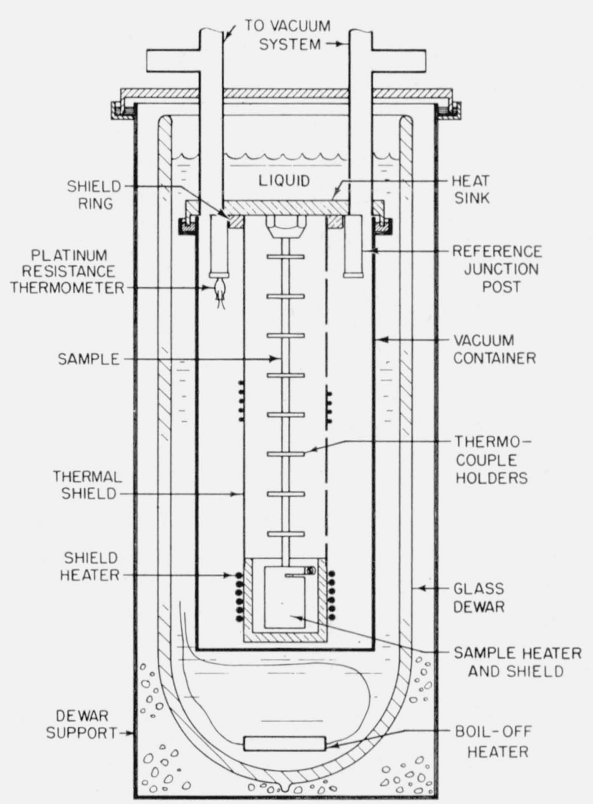

FIGURE 1. Thermal conductivity apparatus.

and power input to the sample, combined with data on the cross-sectional area, permit a calculation of the thermal conductivity of the sample based upon Fourier's equation for steady state, linear heat flow.

The heat sink is maintained at a constant low temperature by a refrigerant, usually a liquefied gas. This refrigerant, poured into the glass Dewar from a central fill tube, surrounds the heat sink and vacuum can. The glass Dewar is supported by a metal container that is, in turn, sealed to a top plate. For temperatures below $80^{\circ} \mathrm{K}$ in the internal apparatus, the Dewar support container is further surrounded by a metal Dewar containing liquid nitrogen, reducing heat conduction down into the colder refrigerant. The associated vacuum, electrical control, and measuring equipment are located on nearby racks and benches. 


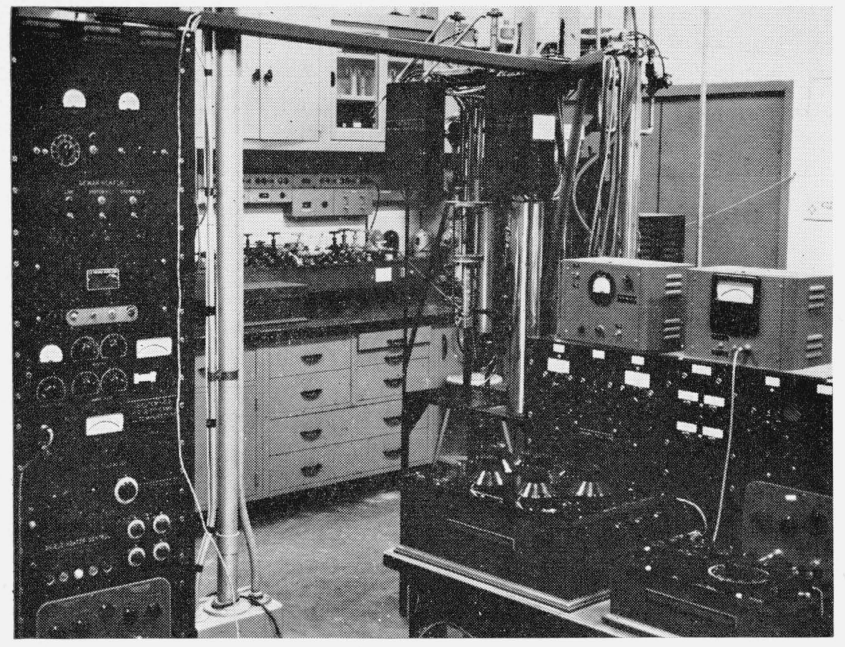

Figure 2. Cryostat and associated equipment.

Figure 2 is a photograph of this assembly. Two similar cryostats are used concurrently to increase the data output.

\subsection{Sample Assembly}

Metal and alloy sample rods are $0.367 \mathrm{~cm}$ in diameter and $23.2 \mathrm{~cm}$ long; plastic samples are 2.54 $\mathrm{cm}$ in diameter and $20.5 \mathrm{~cm}$ long. The metal and alloy rods are turned, then ground to the final diameter with a tolerance of $0.0005 \mathrm{~cm}$. Because of their larger size, the plastics are turned with a tolerance of only $0.001 \mathrm{~cm}$. When required, the sample is annealed after the last grinding operation. Then the thermocouple holders shown in figure 3 are attached, and the holders are placed on the sample with their centers $2.54 \mathrm{~cm}$ apart by means of gage blocks. The thermocouple holders have a thin interior edge that gives, essentially, line contact between the holder and the sample. The actual distances between flat surfaces on the holders are measured by a vernier height gage with an attached precision dial test-indicator. The accuracy is about $0.0005 \mathrm{~cm}$. The average diameters of the sample are measured between the thermocouple holders to about the same accuracy.

The samples are attached to the heat sink by a clamping action for metals, or an internal tapered screw thread for plastics as is shown in figures 1 and 3. The details of the sample heaters are also included in figure 3 . The heater for a metal sample is slipped onto the rod and clamped with a small screw. In order to improve the thermal contact, mercury is placed in the hole that surrounds the lower end of the metal sample. The heater for plastic samples is screwed on so that it presses against a large copper washer used to increase the heater-to-sample contact area. Both heater blocks made of copper are wrapped with No. 36 or 40 AWG constantan heater wire in a single layer held in place with G. E. 7031
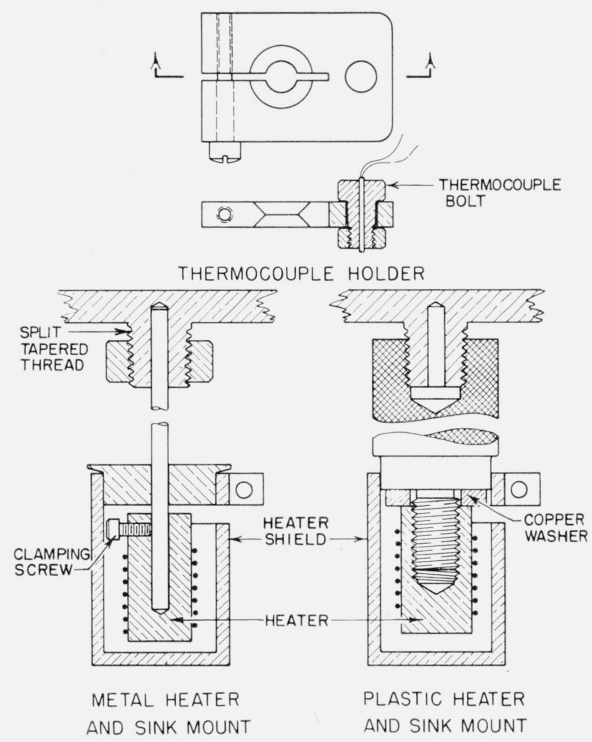

Figure 3. Heaters and thermoccuple holders.

air-drying varnish. The sample heaters have a resistance ranging from 175 to $500 \mathrm{ohms}$ for metals, and from 500 to 1,000 ohms for plastics. The power measuring circuits limit the current to a maximum of about $100 \mathrm{ma}$, and the voltage to a maximum of about $16 \mathrm{v}$. A small sample heater shield is attached to the sample by a split collet-and-screw clamp, so reducing radiation losses. These shields are covered on all exposed surfaces with aluminum foil to further reduce radiation.

\subsection{Thermal Shielding, Tempering, and Controls}

Several factors, such as gas conduction, radiation, and wire conduction, can lead to consistent errors in the experimental determinations of thermal conductivity. Heat losses by gas conduction or convection are reduced to an insignificant amount by baking out and evacuating the system to at least $10^{-5} \mathrm{~mm}$ of $\mathrm{Hg}$ while the system is at room temperature. Cooling of the system during experimental runs further reduces the residual gas pressure.

Heat losses by radiation and lead wire conduction are greatly reduced by surrounding the sample with a cylindrical thermal shield as shown in figure 1 . The bottom, middle, and top of the shield are held at approximately the same temperature as the sample at the corresponding elevation. To provide a good thermal contact to the heat sink when the shield is bolted in place, the upper shield fastening ring is made of copper. The bottom of the shield is also made of copper to provide a nearly isothermal region surrounding the sample heater. The longer intermediate section of the shield is made of thin stainless steel to reduce the power input necessary to maintain any given temperature gradient. The shield is split in two, vertically, to allow rotation of the front half during the mounting and assembling of the sample. 
The two halves are bolted together near the bottom, and a copper bar is soldered across the bottom to improve thermal contact. A mounted sample and the split shield are shown in figure 4 .

Three heaters are wrapped on the shield to supply power necessary to maintain the proper thermal gradient. The main shield heater, wrapped back and forth across the bottom copper section, is made of $32 \mathrm{AWG}$ constantan wire and has about $100 \mathrm{ohms}$ resistance. The other two heaters at the midpoint and near the top, made of $45 \mathrm{AWG}$ constantan, have about 300 ohms resistance. All three are kept in place with air-drying varnish. All wires connected to the sample pass through the shield and are cemented to it at areas close to the same temperature as that of their point of contact on the sample. These wires are 10 to $20 \mathrm{~cm}$ in length between the sample and shield.

The temperature difference between the sample heater and a horizontally opposed point on the lower copper section of the shield is measured with a goldcobalt versus copper thermocouple. Potential differences of about $0.1 \mu \mathrm{v}$ can be detected, corresponding to from $0.02^{\circ} \mathrm{K}$ at liquid helium temperatures, to $0.002^{\circ} \mathrm{K}$ at room temperature. Similar differential thermocouples are at midpoint and near the top.

The thermal electromotive forces developed across the middle and top differential thermocouples are measured with a d-c amplifier-galvanometer. To bring about thermal balance between the shield and sample, as indicated by null readings on the two upper differential thermocouples, the a-c voltages supplied to the heaters at the middle and top of the shield are adjusted manually. If the shield is hotter than the sample at either one of the two upper points, there is no way to obtain thermal balance. 'The lower, or main, differential thermocouple is the sensing device for a heater servomechanism. The approximately correct voltage for the bottom shield heater is adjusted manually by varying two autotransformers in series.

Heater and thermocouple wires are wrapped back and forth upwards on the shield for thermal tempering and then are wound on the posts attached to the heat sink. Two of the six cylindrical copper posts are shown in figure 1. The winding on the posts allows most of the heat conducted down the wires from above to be shorted to the liquid bath. One of the posts serves as a reference junction block for the eight measuring thermocouples; another is used to hold a platinum resistance thermometer that indicates the reference temperature. The others are used for thermal tempering only.

The heat sink and posts are machined from freecutting leaded copper, assuring both good conductivity and machinability [6]. The vacuum chamber cylinder is soldered onto a lip of the heat sink with Rose's Alloy. Two thin stainless steel tubes serve as mechanical support for the internal apparatus, as exit tubes for the wires, and as pumping lines for evacuation of the inside chamber.

For measurements at liquid nitrogen, hydrogen, or helium temperatures, the entire apparatus is sur-

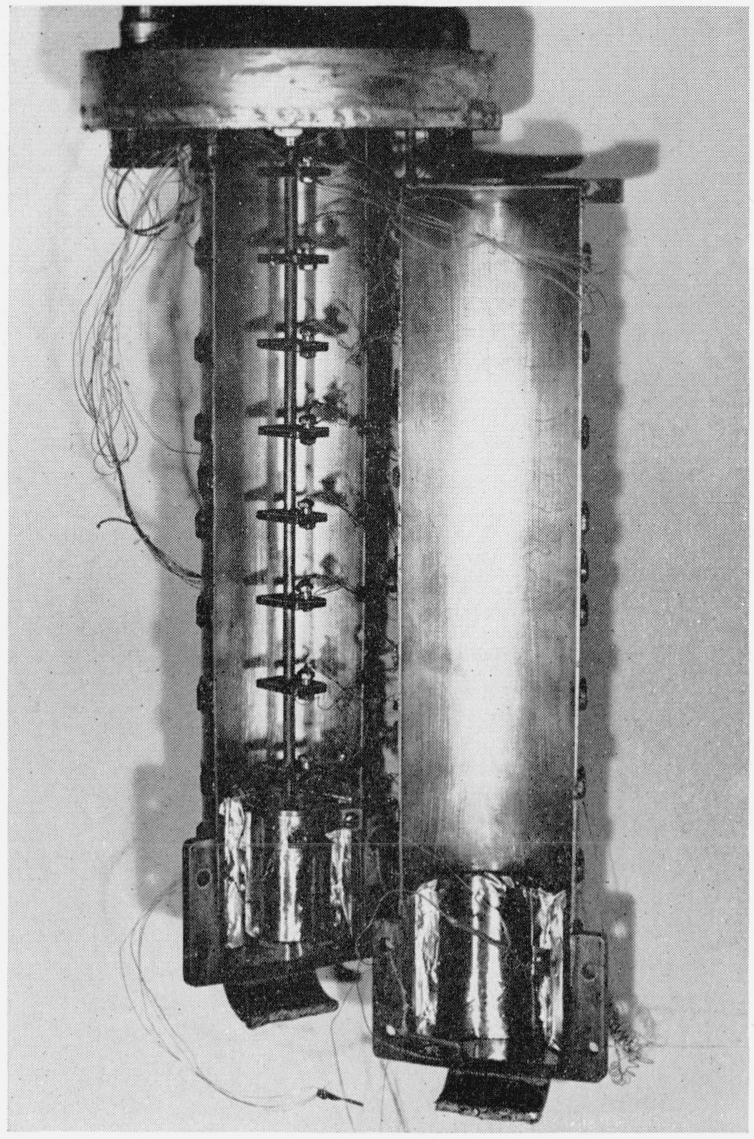

Figure 4. Mounted sample.

rounded by liquid nitrogen in a large metal Dewar. To further reduce heat transfer to the internal apparatus, all the wires are again tied down thermally to a copper post inserted into the pumping line just above the top plate. The wires are brought out from the vacuum space through a hard wax seal. Near the top of the apparatus is a thermally insulated junction box where the various small wires from the cryostat are soldered to larger copper wires, usually $22 \mathrm{AWG}$, which lead to the control panel and measuring instruments.

\subsection{Measuring Apparatus}

The reference junction temperature for the thermocouples is assumed to be the same as that of the thermometer post. The measuring thermometer is a strain-free capsule platinum resistor with an ice point resistance of about $25 \mathrm{ohms}$, which is embedded in one of the posts attached to the heat sink. The resistance of this thermometer, calibrated down to $12^{\circ} \mathrm{K}$ by the Temperature Measurements Section of the Bureau, is measured on a five-dial Mueller bridge. When actual readings are not being taken, this thermometer is switched to a resistance recorder. This switching allows a drift of the heat sink tem- 


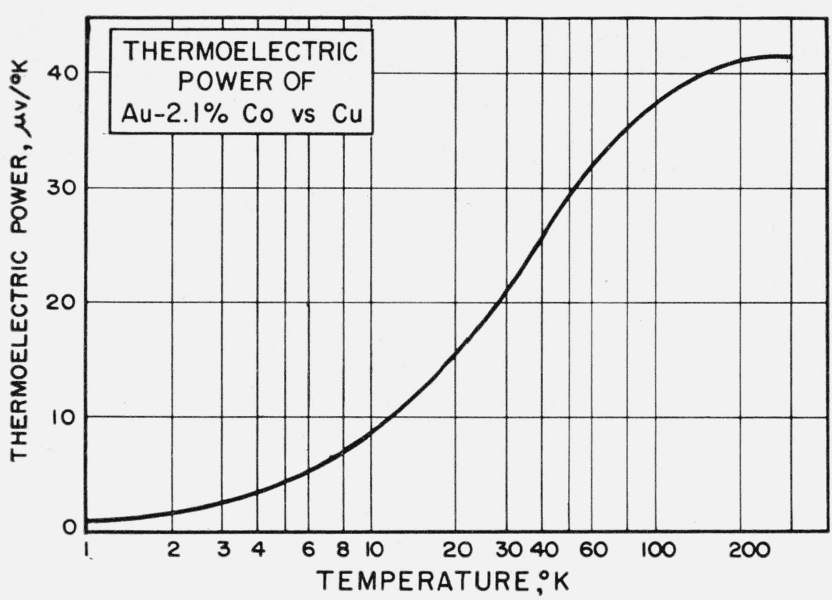

Figure 5. Thermocouple calibration.

perature to be observed without continual manual measurements. With liquid helium, the temperature of the heat sink is obtained from a reading of the barometric pressure by using the $1955 \mathrm{~d}$ compromise temperature scale [2].

Wire of 5-mil diameter, composed of gold with 2.1 atomic percent of cobalt, is used for the common element of the eight thermocouples [1]. The common reference junction is on one post of the heat sink. The external common lead and the separate wires going to each thermocouple junction on the sample are standard thermocouple copper. The thermocouple combination was calibrated in a separate apparatus. Different spools of gold-cobalt wire with the same nominal composition have been found to vary in sensitivity by about 5 to 10 percent. A graph of the sensitivity of the spool that was used is shown in figure 5. Each thermocouple junction has one separate external lead and is measured with reference to the common junction at the heat sink. The junctions are electrically insulated by epoxy resin from the thermocouple bolt and, therefore, from the holder, sample, and ground. The thermocouple leads are soldered to larger wires at the junction box. The voltages are read on a 5 -dial Wenner potentiometer, usually to $0.01 \mu \mathrm{v}$.

A determination of the power to the sample heater is obtained by measuring the d-c voltage and current. Figure 6 shows the circuit diagram. The total current passes through a calibrated 1-ohm NBS-type resistor, $R_{c}$; the voltage developed across it is measured on a Rubicon type- $\mathrm{B}$ potentiometer. The effective current passes through the sample heater $R_{s}$ and the lead resistances $r_{h}$. The currents range from several milliamperes to about 100. The voltage across the heater is divided across three calibrated resistors, arranged to give either 2 to 1 or 11 to 1 voltage division. Resistor $R_{3}$ is 10 $\mathrm{K}$ ohms; $R_{\mathrm{p}}$ is either $10 \mathrm{~K}$ ohms for 2 to 1 voltage divider ratio, or $1 \mathrm{~K}$ ohm for 11 to 1 ratio. The voltage, measured on the same potentiometer as the current, ranges from 1 to about $16 \mathrm{v}$. The four calibrated resistors are in an oil bath maintained at $25^{\circ} \mathrm{C}$ by a commercial mercury thermostat and infrared heating unit.

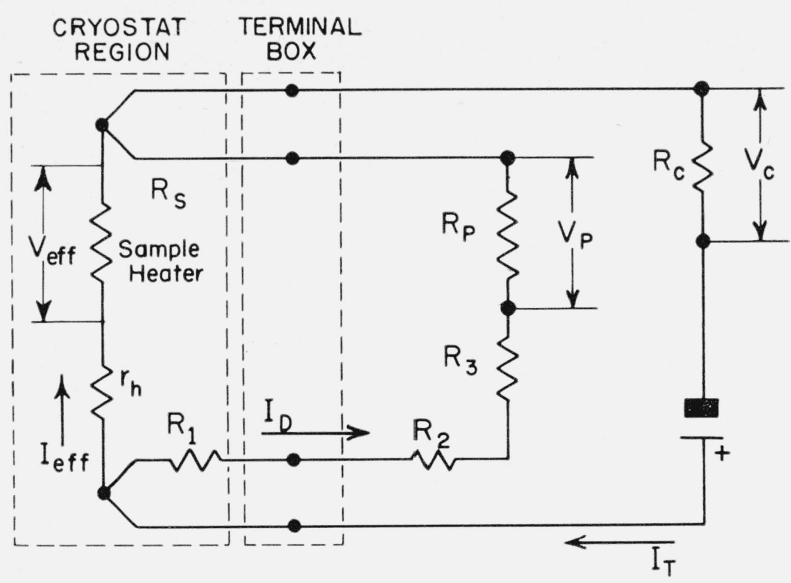

Figure 6. Heater circuit.

Galvanometer unbalance voltages from the bridge and two potentiometers are directed to a commercial d-c breaker amplifier rather than the more commonly used moving-coil galvanometers. The input selector switch to the amplifier can be set to any of the galvanometer terminals of the bridge or potentiometers, or to some of the differential thermocouples between the sample and shield. The high sensitivity and several-million-fold amplification of the breaker amplifier allow interpolation one figure beyond the last dial setting on either the Mueller bridge or the Wenner potentiometer. The signal from the amplifier is switched to a $\mathrm{l}-\mathrm{v}$, center zero, d-c voltmeter for precise readings, or to a slow-speed recorder for following temperature drifts or approach to equilibrium.

Standard low-level electric techniques have been used: that is, all wires are shielded; a special common ground wire is used; the bridges are on insulated ground plates; and all terminals, switches, batteries, and standard cells are thermally insulated.

\subsection{Auxiliary Equipment}

A photograph of the equipment is shown in figure 2 . The vacuum control unit is behind the cryostat; the power panels and control are on the left. The sample space is evacuated through nominal 1-in. tubes by an oil diffusion pump. Between the system and the diffusion pump is a metal cold trap that can hold liquid nitrogen over a weekend without refilling. Between the diffusion pump and the rotary mechanical pump is a 2-liter reservoir that, with its valve to the mechanical pump closed, can normally hold the diffusion-pump output for $4 \mathrm{hr}$ before the pressure builds up to $100 \mu$. In parallel with the cold trap, diffusion pump, and reservoir, is a bypass line for pumping the system down initially without breaking the vacuum in the cold trap or diffusion pump. The pressure in the sample system is measured with a commercial ion gage when it is below $1 \mu$, and with a thermocouple gage when above that. The pressure in the reservoir, that is, the back pressure of the diffusion pump, is measured on another thermo- 
couple gage system that has shutoff relays controlling the diffusion pumps and ion gage. Whenever the back pressure goes above $100 \mu$, an alarm light turns on, and the power to the diffusion pump and ion gage turns off.

The liquid refrigerant can be either maintained at a reduced pressure by a high-capacity mechanical pump, or vented - directly to an explosion-proof exhaust system. These vent lines are necessary to reduce the explosion danger during liquid hydrogen tests. The vapor space over the liquid is connected to a mercury manometer, permitting measurement of the vapor pressure of the bath.

The power panel supplies both a-c and d-c voltage to the heaters and electronic equipment. The d-c voltage is obtained from a large bank of nickelcadmium batteries in a nearby room. The current through the main sample heater, which is adjusted by four variable decade resistors, is read preliminarily on a precision multirange milliammeter. The a-c power is obtained from a $110-v$ regulated power supply. Power to each of the shield heaters is controlled by two autotransformers in series. The a-c power supply also furnishes voltage to a millivolt recorder, breaker amplifier, and the main shield heater servomechanism.

\subsection{Measurement Techniques}

For each metal sample, the thermocouple holders are mounted in a temperature-controlled shop room, where the dimensions are also measured. After these preliminary measurements, the sample is mounted in the heat sink and the heaters and thermocouples are attached. The sample is not heated or soldered after assembly of the thermocouple holders, and it is subjected to as little mechanical strain as possible during insertion into the cryostat. After this assembly, the shield is closed and bolted; then the vacuum can is soldered into place with Rose's Alloy. The sample space and tubes are evacuated and baked out for several days, usually over a weekend. The liquid refrigerants are not placed into the glass Dewar until the pressure in the cryostat is down to at least $10^{-5} \mathrm{~mm}$ of $\mathrm{Hg}$.

Experimental runs are usually begun at liquid helium temperatures, after precooling first with nitrogen, then with hydrogen; and then the runs are continued at the hydrogen triple point, hydrogen boiling point, nitrogen triple point, nitrogen boiling point, carbon dioxide sublimation point, and then ice point. Normally, about six series of measurements at various temperature gradients are made in each temperature range. Two sets of measurements are made for each gradient to insure against reading errors or lack of steady state. The over-all temperature differences across the sample vary from $1^{\circ} \mathrm{K}$ to a sufficiently large temperature necessary to overlap readings in the next higher temperature range. The approach to steady-state conditions is observed by amplifying the unbalance emf from the Wenner potentiometer with the input switch set for the lowest thermocouple. The amplified signal is switched to the millivolt recorder, and, when this thermo- couple emf no longer drifts with time, precise measurements of the voltage, current, and temperatures are begun. Measurements are also made at each sink temperature with no power input and with the normal vacuum space filled with helium exchange gas at a pressure of several hundred microns. Because the apparatus should be isothermal under these conditions, these readings give the "zero" corrections for the thermocouples. These corrections are attributed primarily to inhomogeneous emf's and are assumed to be constant for a particular sink temperature.

Subsidiary measurements are made afterwards on a short section of the sample. They include mounting and polishing of the metal for photomicrographs of grain structure, measurement of hardness, measurement of density, and spectrographic or chemical analyses.

\section{Analyses of Data}

\subsection{Basic Calculations}

The fundamental equation of heat flow is utilized in its differential form:

$$
\dot{Q}=-\lambda A \frac{d T}{d x}
$$

where $\dot{Q}$ is the heat flux, $\lambda$ is the thermal conductivity, and $A$ the cross-sectional area. Because $\dot{Q}$ and $A$ are essentially constants of the sample for each run,

$$
\lambda=\frac{d(-\dot{Q} x / A)}{d T} .
$$

Because we do not measure the temperature directly, but rather the thermocouple voltage $E$, we rearrange the equation to

$$
\lambda=\frac{d(-\dot{Q} x / A)}{d E} \frac{d E}{d T}
$$

where $d E / d T$ is the thermoelectric power of the thermocouple at the given voltage $E$ and for the given reference temperature.

Several corrections to a straightforward calculation of the heat power of the sample heater are necessary. The various components of the power circuit are shown in figure 6 . Basically, the power is the product of the voltage and current through the sample heater. The effective current through the heater is given by

$$
I_{e f f}=I_{T}-I_{D}
$$

where $I_{T}$ is the total current and $I_{D}$ is the current through the divider circuit. The total current is given by the measured voltage $V_{C}$ across the standard resistor divided by its resistance $R_{C}$. Similarly, the divider current is given by the measured divider voltage $V_{P}$ divided by the resistance of the particular resistor $R_{P}$. The effective current is then

$$
I_{e f f}=\frac{V_{C}}{R_{C}}-\frac{V_{P}}{R_{P}}
$$


The effective voltage is

$$
V_{\text {eff }}=\rho V_{P}-r_{h} I_{e f f},
$$

where $r_{h} I_{\text {eff }}$ is the temperature-dependent voltage developed across the heater leads between the shield and the sample, and $\rho$ is the voltage divider ratio. The ratio is given by

$$
\rho=\frac{R_{1}+R_{2}+R_{3}+R_{P}}{R_{P}}
$$

where $R_{1}$ and $R_{2}$ are lead resistances ( $R_{1}$ is temperature dependent), $R_{3}$ is a standard $10 \mathrm{~K}$ ohm resistor, and $R_{P}$ is either $1 \mathrm{~K}$ ohm or $10 \mathrm{~K}$ ohm.

The cross section and distances along the sample are measured at room temperature. These dimensions are corrected for thermal contraction at each sink temperature range whenever thermal expansion data are available. A preliminary plot is made of $\dot{Q} x / A$ against $E$ to check for points that are considered to have too large a deviation.

For the acceptable runs, the $\dot{Q}_{x / A}$ and $E$ are tabulated, and the values are processed by an IBM 650 digital computer. The program fits the $\dot{Q} x / A$ data by a least squares method to polynomials to the first, second, third, and fourth degree in $E$. Generally speaking, the first-degree polynomials give the best results for small temperature gradients, and the fourth-degree polynomials give the best for the large gradients. The computer is then programed to take the derivative of each of the polynomials, and then to multiply by the thermoelectric power of the thermocouples at each temperature. The final result is the thermal conductivity at 1-deg intervals based upon each of the above polynomials.

Thermal conductivities as a function of temperature for each run are then plotted on a large scale graph. Average or best curves are drawn through the many points for each range, and the various ranges are connected smoothly to give the complete graph.

\subsection{Estimation of Errors}

Three main sources of error appear in the apparatus described here: (1) Calibration of the thermocouples, (2) placement of the thermocouple holders, and (3) thermal contact resistance between the thermocouple and the sample. The first can be improved by a more complete and accurate thermocouple calibration. A new series of thermocouple calibrations to improve the range and accuracy of the data has been started in thermometry calibration apparatus. The second source of error has been practically eliminated because of better control of the dimensions and placement of the holders. The third source of error can be reduced by increasing the length of the thermocouple lead wire between the shield and the sample, and by obtaining better contact between the thermocouple bolt and its holder.
Even after improvement, the first and third will probably remain the main sources of error. The thermocouple measurements can be improved, but appreciable errors will still come from chemical inhomogeneities, mechanical strains, uncertainty in calibration, reduced sensitivity at the lowest temperatures, and thermal emf in the external circuitry. The thermocouple error is estimated to be less than 1 percent over most of the temperature range. This error will be systematic and cannot be estimated or eliminated by statistical methods. The error caused by thermal contact resistance can be estimated by deliberately holding the thermal shield hotter or colder than the sample.

Except for gas conduction and radiation at high temperatures, the other sources of error have been reduced to an insignificant amount, considering the larger errors mentioned above. Errors in the crosssectional measurements are probably about 0.2 percent; inaccuracy in locating the actual contact point between the thermocouple holder and sample is about the same. Errors due to radiation below $100^{\circ} \mathrm{K}$ and conduction along lead wires were calculated to be below 0.1 percent. To check these radiation losses at higher temperatures, several trial runs with different gradients were made at the ice point. Apparently, for metals, an error of about 1 to 2 percent exists in the readings at that temperature as disclosed by a systematic dependence of the apparent conductivity on the gradient. Several runs were made with a residual helium gas pressure of approximately $1 \times 10^{-5} \mathrm{~mm} \mathrm{Hg}$ within the system. These runs differed by about 3 percent for most temperature ranges.

Experimental runs in each temperature range have been analyzed for internal consistency. The deviations from an average curve are usually less than 1 percent in the liquid helium and hydrogen ranges, and less than $\frac{1}{2}$ percent at liquid nitrogen temperatures and above. These inconsistencies are apparently due to the combined result of the various errors mentioned above, the one exception being thermocouple calibration error, which is systematic. The inaccuracies increase if the sample has either a very high or a very low conductivity. The range of usable sample diameters is not as great as the variation in conductivity; therefore, at both extremes of conductivity, optimum measuring conditions cannot be obtained. Recent measurements on a very pure copper sample that had a conductivity maximum over $100 \mathrm{w} / \mathrm{cm}{ }^{\circ} \mathrm{K}$ gave inconsistencies of about 5 percent between $4^{\circ} \mathrm{K}$ and $60^{\circ} \mathrm{K}$. Measurements on very low conductivity alloys or plastics have about this same inaccuracy, and, in addition, are usually not reliable above $100^{\circ} \mathrm{K}$ because of the increasing significance of radiation exchange between the sample and shield.

Many control experiments have been carried out to test the self-consistency of the apparatus. Many of the runs overlap in temperature range. For example, some of the runs were carried out with the heat sink at $4^{\circ} \mathrm{K}$ and the bottom of the sample 
around $25^{\circ} \mathrm{K}$, thus overlapping runs made with the sink at $19.7^{\circ} \mathrm{K}$. The conductivity curves from the lower ranges fit smoothly with the curves at the higher temperatures. Apparent conductivities from runs with different thermal gradients on the sample usually agree within the scatter normally characteristic of a single run. Various runs with different size samples and holders gave consistent results.

\section{Results}

\subsection{Polytetrafluoroethylene}

The graph of thermal conductivity of this plastic is given in figure 7 . The estimated inaccuracy of the results is about 10 percent. The sample was extruded and had a density of $2.218 \mathrm{~g} / \mathrm{cm}^{3}$. Thermal contraction was calculated by using the data of Quinn, et al. [7] for the higher temperatures, and the data of Head and Laquer [3] for the low temperatures [4].

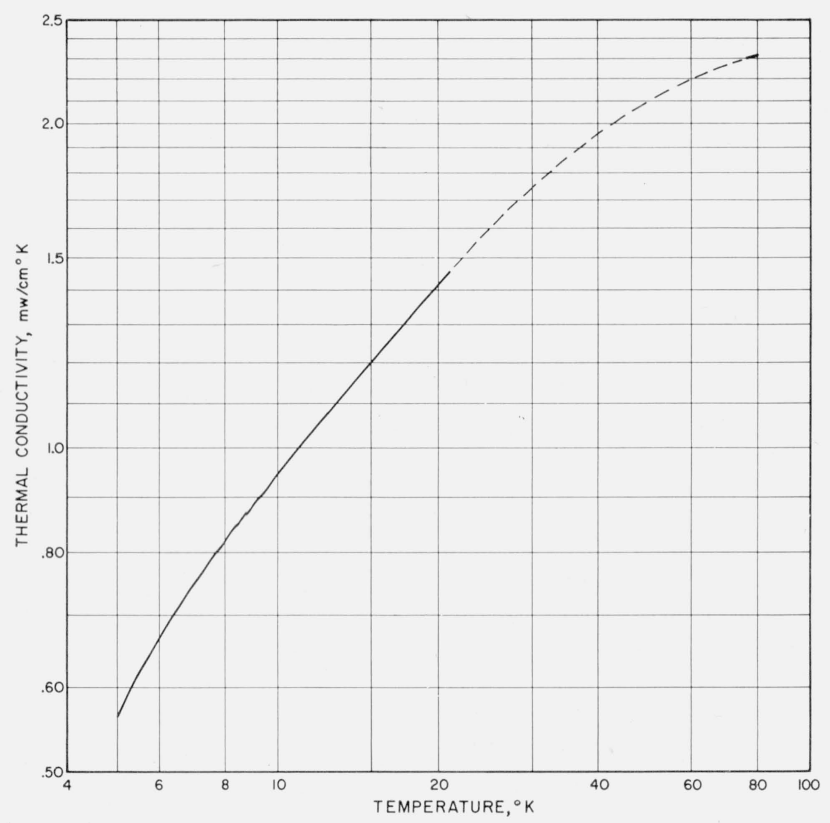

FIGURE 7. Thermal conductivity of polytetrafluoroethylene. (The dashed lines indicate interpolated regions.)

\subsection{Coalesced Copper}

The graph of the thermal conductivity of this high-purity commercial copper is given in figure 8 . The estimated inaccuracy of these results ranges from 2 to 5 percent. The sample had a mill specification giving the impurities by weight as $13 \mathrm{ppm}$ $\mathrm{O}_{2} ; 8 \mathrm{ppm} \mathrm{Pb} ; 7 \mathrm{ppm} \mathrm{Ni}$; less than $5 \mathrm{ppm}$ each of $\mathrm{Fe}$,

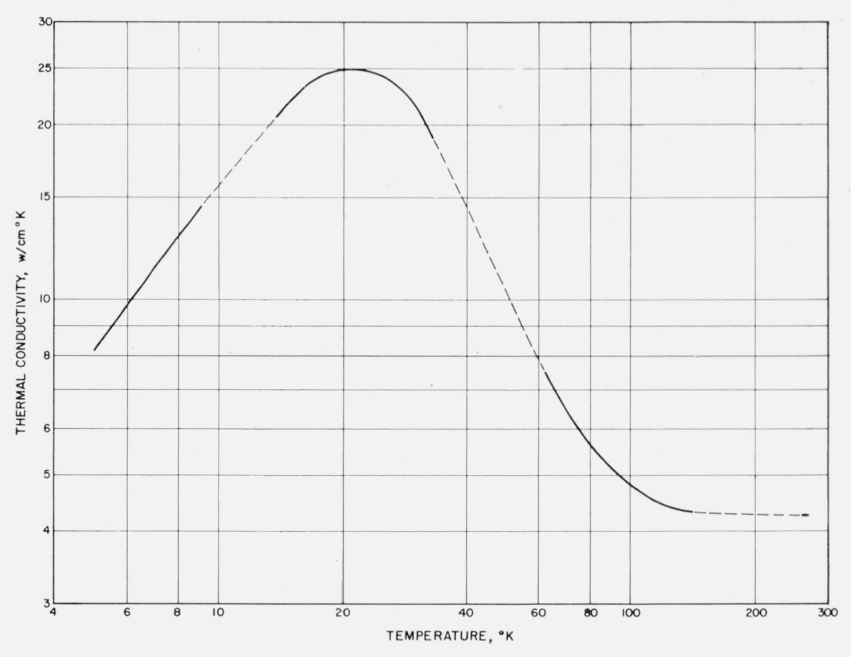

Figure 8. Thermal conductivity of coalesced copper. (The dashed lines indicate interpolated regions.)

As, and Sb; 2 ppm Sn; less than 1 ppm Te and $\dot{A} g$; and less than $0.5 \mathrm{ppm} \mathrm{Bi}$. The sample was annealed $4 \mathrm{hr}$ at $400^{\circ} \mathrm{C}$, cooled slowly to $200^{\circ} \mathrm{C}$, and then kept at $200^{\circ} \mathrm{C}$ for $8 \mathrm{hr}$. During the entire heating cycle, the sample was kept in a flowing helium atmosphere. The density of the sample was $8.90 \mathrm{~g} / \mathrm{cm}^{3}$, and its hardness on the Vickers diamond point system with a $10 \mathrm{~kg}$ weight was 54.1 in the longitudinal section, and 48.8 in the transverse.

The authors thank R. J. Corruccini, W. B. Hanson, and R. B. Scott for advice and assistance during the progress of this project, and G. A. Yates of the Phelps Dodge Copper Products Corp. for the generous supply of samples and fabrication information.

\section{References}

[1] G. Borelius, W. H. Keesom, C. H. Johansson, and J. O. Linde, Commun. Phys. Lab. Univ. Leiden, No. 206b.

[2] J. R. Clement, liquid helium vapor pressure-temperature scale. Distributed by the Naval Research Laboratory as an informal report (1955).

[3] E. L. Head and H. L. Laquer, Low temperature thermal expansion of various materials, Available from Office of Technical Services, AEC D-3706.

[4] Richard K. Kirby, J. Research NBS 5\%, 91 (1956) Received after completion of the data analysis.

[5] J. L. Olsen and H. M. Rosenberg, Adv. Physics 2, 28 (1953).

[6] R. L. Powell and D. O. Coffin, Rev. Sci. Inst. 26, 516 (1955).

[7] F. A. Quinn, D. E. Roberts, and R. N. Work, J. Appl. Phys. 22, 1085 (1951).

Boulder, Colo., December 8, 1956. 